\title{
Effect of adenosine on GLAST expression in the retina of a chronic ocular hypertension rat model
}

\author{
ZI-JIAN YANG and YI-SHENG ZHONG \\ Department of Ophthalmology, Ruijin Hospital Affiliated Medical School, \\ Shanghai Jiaotong University, Shanghai 200025, P.R. China
}

Received July 28, 2014; Accepted April 2, 2015

DOI: $10.3892 /$ etm.2015.2607

\begin{abstract}
This study was performed to evaluate the effect of adenosine and an adenosine receptor antagonist on the expression of the L-glutamate/L-aspartate transporter (GLAST) in the retina of a chronic ocular hypertension $(\mathrm{COH})$ rat model. $\mathrm{COH}$ models were established via the cauterization of three episcleral veins. Measurements of the intraocular pressure of the right eye ( $\mathrm{COH}$ eye) were taken weekly by a handheld digital tonometer. A total of $10 \mu \mathrm{M}$ adenosine or $10 \mu \mathrm{M}$ adenosine $+100 \mathrm{nM}$ SCH442416 solution $(2 \mu \mathrm{l})$ was injected into the rat vitreous space. The reverse transcription-quantitative polymerase chain reaction, western blotting and immunohistochemistry were used to detect GLAST expression. Compared with the $\mathrm{COH}$ group, GLAST mRNA expression was decreased by $33.6 \%$ in the group treated with adenosine $(n=6, P=0.020)$ and was increased by $159.6 \%$ in the group treated with $\mathrm{SCH} 442416(\mathrm{n}=6, \mathrm{P}=0.001)$. Administration of adenosine decreased GLAST protein expression by $34.7 \%$ $(n=6, P<0.001)$, while treatment with the adenosine $A_{2 A}$ receptor antagonist $\mathrm{SCH} 442416$ increased GLAST protein expression by $48.3 \%$ compared with the control $\mathrm{COH}$ group $(n=6, P<0.001)$. Immunohistochemical experiments showed that administration of adenosine decreased GLAST protein expression, as compared with expression in the control $\mathrm{COH}$ rat retina. Administration of SCH442416 markedly increased GLAST protein expression. The results of the present study may provide a novel method for retinal neuron protection.
\end{abstract}

\section{Introduction}

Adenosine is an endogenous bioactive substance widely present in the intracellular and extracellular fluid in mammals. Almost

Correspondence to: Professor Yi-Sheng Zhong, Department of Ophthalmology, Ruijin Hospital Affiliated Medical School, Shanghai Jiaotong University, 197 Ruijin No. 2 Road, Shanghai 200025, P.R. China

E-mail: yszhong68@126.com

Key words: chronic ocular hypertension, adenosine, adenosine receptor antagonist, intraocular pressure, GLAST, Müller cell all cells, including Müller cells, can release adenosine. Müller cells release adenosine through a calcium-independent facilitated transport method $(1,2)$. Adenosine is a local hormone that is present at a low level in physiological conditions, normally at a concentration of 20-200 $\mathrm{nM}$. Tissue hypoxia or ischemia can lead to adenosine triphosphate breakdown and the increased generation of adenosine (3). Adenosine, which exerts anti-inflammatory effects, is released from the retina and has an important role in pathological conditions, such as ischemia-hypoxia and glaucoma (4). To a certain extent, adenosine protects neurons from glutamate toxicity by suppressing excitatory neurotransmission (5).

Glutamate is a neurotransmitter that can be found in brain tissue and the retina of the eye (6). In conditions of depolarization, Müller cells release glutamate and the increased non-vesicular release of glutamate causes excitotoxic damage to neurons (7). The L-glutamate/L-aspartate transporter (GLAST) is a primary glial enzyme in the clearance of extracellular glutamate in physiological conditions (8). In pathological conditions, the functional downregulation of GLAST induces an increased glutamate concentration in the retina; however, the association between the high concentration of adenosine and GLAST downregulation in the retina is not clear. The aim of the present study, therefore, was to explore the effects of adenosine and an adenosine receptor antagonist, $\mathrm{SCH} 442416$, on rat retina GLAST expression.

\section{Materials and methods}

Rat chronic ocular hypertension $(\mathrm{COH})$ models. All experimental procedures described were in accordance with the National Institutes of Health Guidelines for the Care and Use of Laboratory Animals. The present study was approved by the laboratory animal ethics committee of Ruijin Hospital (Shanghai, China). Male Sprague Dawley rats (weight, 200-250 g) were purchased from Shanghai SIPPR-BK Laboratory Animal Co., Ltd. (Shanghai, China), and housed in an air-conditioned animal room at $\sim 23^{\circ} \mathrm{C}$ under a 12 -h light/dark cycle. All efforts were made to minimize the suffering of the rats in this study. Prior to all surgeries and procedures, the animals were anesthetized with an intraperitoneal injection of xylazine and ketamine hydrochloride $(7.4 \mathrm{mg} / \mathrm{ml}$ and $5 \mathrm{mg} / \mathrm{kg}$, respectively) (Jiangsu Hengrui Medicine Co., Ltd., Lianyungang, China). Two drops of $0.3 \%$ Ocuflox ${ }^{\circledR}$ solution (Santen Pharmaceutical Co., Ltd., 
Osaka, Japan) were topically applied prior to and following the eye surgeries to prevent infection.

The rat $\mathrm{COH}$ model was reproduced following a procedure described in previous studies $(9,10)$. An increase in the intraocular pressure (IOP) was induced in 60 rats by cauterizing three episcleral veins in the right eye of each rat. Briefly, three veins (including two episcleral veins beside the superior rectus muscle and one episcleral vein beside the lateral rectus muscle) were separated and cauterized precisely to avoid nearby tissue damage. The right eyes of a further 6 rats were subjected to a sham procedure. IOP measurements were taken weekly following surgery at the same time in the morning. Proxymetacaine hydrochloride solution (Alcon Laboratories, Inc., Fort Worth, TX, USA) was used topically prior to the IOP measurements, which were conducted with a handheld digital tonometer (TonoPen ${ }^{\circledR}$; Mentor Ophthalmics, Inc., Norwell, MA, USA). The probe of the TonoPen was applied vertically to the cornea surface, and touched the cornea surface with the same force in all measurements. If the confidence interval was $\geq 95 \%$, numerical values were accepted. Ten numerical values were recorded in each eye.

Drugs and intravitreal injection. Prior to injection, the rat pupil was dilated with a tropicamide drop (Qianjiang Pharmaceutical Co., Ltd., Qianjiang, China). A total of $10 \mu \mathrm{M}$ adenosine (Sigma-Aldrich, St. Louis, MO, USA) or $10 \mu \mathrm{M}$

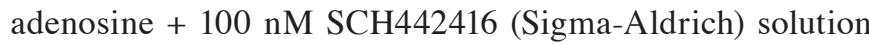
( $2 \mu \mathrm{l}$ ) was injected into the rat vitreous space ( $\mathrm{n}=6$ rats/group). Adenosine or adenosine + SCH442416 were first dissolved in dimethyl sulfoxide (DMSO) and then diluted by double-distilled $\mathrm{H}_{2} \mathrm{O}$ (final concentration DMSO, 5\%). Under a stereoscopic microscope, a microinjector (Hamilton Robotics, Inc., Reno, NV, USA) was inserted $2 \mathrm{~mm}$ behind the temporal limbus and directed toward the optic nerve to inject solution into the vitreous space. Eyes that received only an injection of vehicle solution in the same manner served as controls.

Reverse transcription-quantitative polymerase chain reaction $(R T-q P C R)$ analysis. First, total RNA, isolated by TRIzol $^{\circledR}$ (Invitrogen Life Technologies, Carlsbad, CA, USA), was reverse-transcribed into cDNA. The PCR solution contained $2 \mu \mathrm{l} \mathrm{cDNA}$, the specific primer set ( $1 \mu \mathrm{M}$ each) and $11.5 \mu \mathrm{l}$ QuantiTect SYBR ${ }^{\circledR}$ Green PCR Master Mix from the QuantiTect SYBR Green PCR kit (Qiagen, Hilden, Germany) in a final volume of $20 \mu \mathrm{l}$. The following primer pairs (Invitrogen Life Technologies) were used: GLAST sense, 5'-CCTATGTGGCAGTCGTTT-3' and anti-sense, 5'-CTG TGATGGGCTGGCTAA-3', $\beta$-actin sense, 5'-GCGCTCGTC GTCGACAACGG-3' and anti-sense, 5'-GTGTGGTGCCAA ATCTTCTCC-3'. The PCR cycle conditions were as follows: i) Initial denaturation, one cycle at $94^{\circ} \mathrm{C}$ for $5 \mathrm{~min}$; ii) amplification and quantification, 40 cycles at $94^{\circ} \mathrm{C}$ for $30 \mathrm{sec}, 55^{\circ} \mathrm{C}$ for $30 \mathrm{sec}$ and $72^{\circ} \mathrm{C}$ for $30 \mathrm{sec}$; iii) melting curve, $55^{\circ} \mathrm{C}$ with the temperature gradually increased up to $95^{\circ} \mathrm{C}$.

Western blot analysis. For western blotting, the rat retinas were lysed in radioimmunoprecipitation assay buffer (Beyotime Institute of Biotechnology, Shanghai, China) in the presence of protease inhibitors. The homogenates were centrifuged at $14,000 \times \mathrm{g}$ for $30 \mathrm{~min}$ at $4^{\circ} \mathrm{C}$. The protein concentrations were determined using the bicinchoninic acid method. Equal quantities of protein $(1 \mu \mathrm{g} / \mu \mathrm{l}, 15 \mu \mathrm{l})$ were separated by $10 \%$ SDS-PAGE and transferred to polyvinylidene fluoride membranes. Subsequent to being blocked with 5\% skimmed milk at room temperature for $2 \mathrm{~h}$, the membranes were incubated with rabbit polyclonal antibody against GLAST (1:200 dilution; cat. no. ab416; Abcam, Cambridge, UK) or mouse monoclonal antibody against GAPDH (1:10,000 dilution; cat. no. KC-5G4 KangChen Bio-tech Inc., Shanghai, China) overnight at $4^{\circ} \mathrm{C}$. The membranes were then washed three times with Tris-buffered saline-Tween 20 for $10 \mathrm{~min}$ and incubated with horseradish peroxidase-conjugated goat anti-rabbit secondary antibody (1:2,000 dilution) for $1 \mathrm{~h}$ at room temperature. Images were captured using the ImageQuant ${ }^{\mathrm{TM}}$ Las 4000 mini biomolecular imager (GE Healthcare Life Sciences, Pittsburgh, PA, USA), and the protein bands were quantitatively analyzed with Image-Pro Plus version 6.0.0.260 (Media Cybernetics, Inc., Rockville, MD, USA) image analysis software.

Immunohistochemistry. Rats were perfused with normal saline and $4 \%$ paraformaldehyde (PFA) solution. The right eyeballs were fixed in $4 \%$ PFA solution for $4 \mathrm{~h}$, and then dehydrated with a graded sucrose solution at $4^{\circ} \mathrm{C}$ ( $4 \mathrm{~h}$ in $20 \%$ sucrose solution and overnight in $30 \%$ sucrose solution). The retinas were vertically sectioned at a $7.5-\mu \mathrm{m}$ thickness and then mounted on gelatin-coated slides. Subsequent to being rinsed with $0.01 \mathrm{M}$ phosphate-buffered saline (PBS), the retina slices were blocked in 4\% goat serum (Gibco Life Technologies, Carlsbad, CA, USA), $0.25 \%$ bovine serum albumin (Sigma-Aldrich) and $0.2 \%$ Triton X-100 (Solarbio Science \& Technology Co., Ltd., Shanghai, China) in PBS at room temperature for $2 \mathrm{~h}$, and incubated with goat polyclonal anti-GLAST primary antibody (1:200 dilution; cat. no. sc-7758; Santa Cruz Biotechnology, Inc., Santa Cruz, CA, USA) at $4^{\circ} \mathrm{C}$ for $48 \mathrm{~h}$. Following incubation with the primary antibody, the retina slices were rinsed in 0.01 M PBS and incubated with the fluorescein isothiocyanate-conjugated donkey anti-goat immunoglobulin G secondary antibody (1:100 dilution; Jackson ImmunoResearch Laboratories, West Grove, PA, USA) for $2 \mathrm{~h}$ at room temperature. Finally, the retina slices were mounted with anti-fade mounting medium with DAPI (Vector Laboratories, West Grove, PA, USA), and the immunofluorescence images were visualized with a Zeiss Imager M1 laser-scanning microscope (Carl Zeiss AG, Oberkochen, German) using a 20X objective lens.

Statistics. Data were analyzed using SPSS 19.0 software (IBM SPSS, Armonk, NY, USA) and are presented as the mean \pm standard deviation. The paired-samples Student's t-test or one-way analysis of variance was used to test the data for statistical significance. $\mathrm{P}<0.05$ was considered to indicate a statistically significant difference.

\section{Results}

IOP elevation in the COH models. The mean IOP in the sham-operated animals exhibited no significant difference between the right $(19.03 \pm 0.25 \mathrm{mmHg})$ and left $(19.47 \pm 0.69 \mathrm{mmHg})$ eye in the 8 -week observation period 


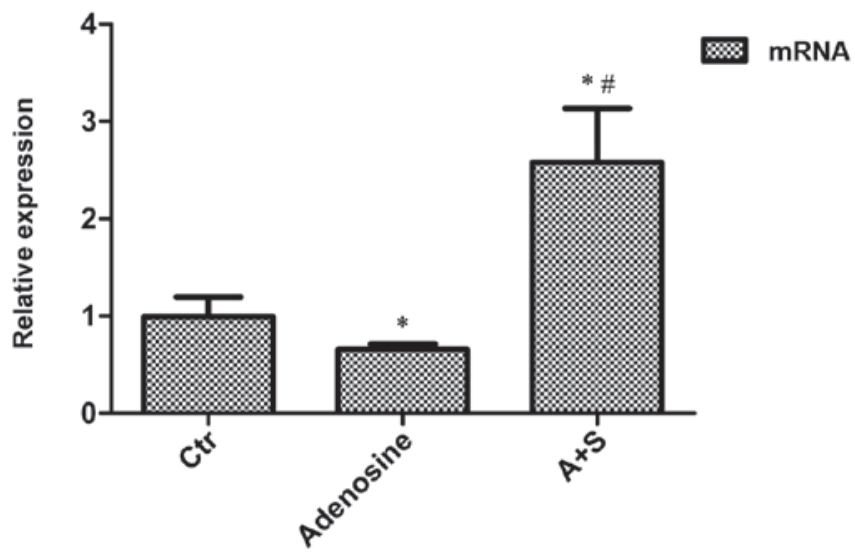

Figure 1. Effects of adenosine and SCH442416 on GLAST mRNA expression in rat models of $\mathrm{COH}$. Adenosine and/or SCH442416 were injected into the rat vitreous space 2 weeks before the surgery. The relative expression of GLAST mRNA in the right eyes of the control (COH), adenosine- and SCH442416-treated rats is shown. Data are presented as the mean \pm standard deviation, $\mathrm{n}=6$. ${ }^{*} \mathrm{P}<0.05$ versus control; ${ }^{\#} \mathrm{P}<0.05$ versus the adenosine group. $\mathrm{A}+\mathrm{S}$, adenosine + $\mathrm{SCH} 442416$; ctr, control; $\mathrm{COH}$, chronic ocular hypertension; GLAST, L-glutamate/L-aspartate transporter.

A

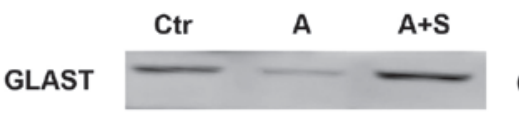

$60 \mathrm{kDa}$

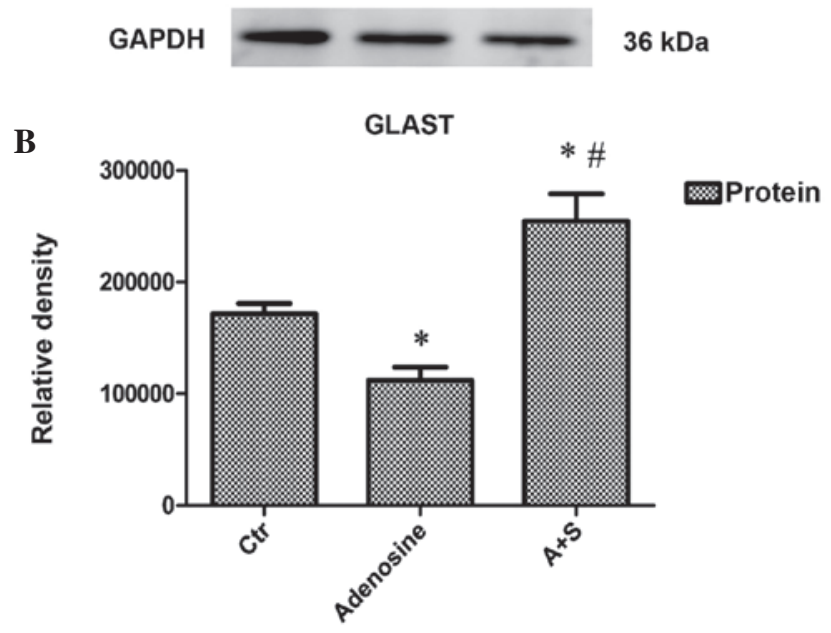

Figure 2. Effect of adenosine and SCH442416 on GLAST protein expression in the retinas of $\mathrm{COH}$ rat models. Adenosine and/or SCH442416 were injected into the rat vitreous space 2 weeks before the surgery. (A) GLAST protein expression in the right eyes of the control $(\mathrm{COH})$, adenosine- and SCH442416-treated rats. (B) Bar chart summarizing the average densitometric quantification of the GLAST western blot bands for the control $(\mathrm{COH})$, adenosine- and SCH442416-treated rats. Data are presented as the mean \pm standard deviation, $\mathrm{n}=6 .{ }^{*} \mathrm{P}<0.05$ versus control, ${ }^{\#} \mathrm{P}<0.05$ versus the adenosine group. $\mathrm{A}+\mathrm{S}$, adenosine $+\mathrm{SCH} 442416$; ctr, control; $\mathrm{COH}$, chronic ocular hypertension; GLAST, L-glutamate/L-aspartate transporter.

$(n=6, P=0.329)$. In the episcleral vein cauterization group the IOP of the right eye showed a significant increase from the first week of the study and continued to show a steady increase until the eighth week. The mean IOP of the right eye in the episcleral vein cauterization group was $27.3 \pm 1.83 \mathrm{mmHg}$, compared with $19.03 \pm 0.25 \mathrm{mmHg}$ in the sham surgery group $(n=6, P=0.001)$.
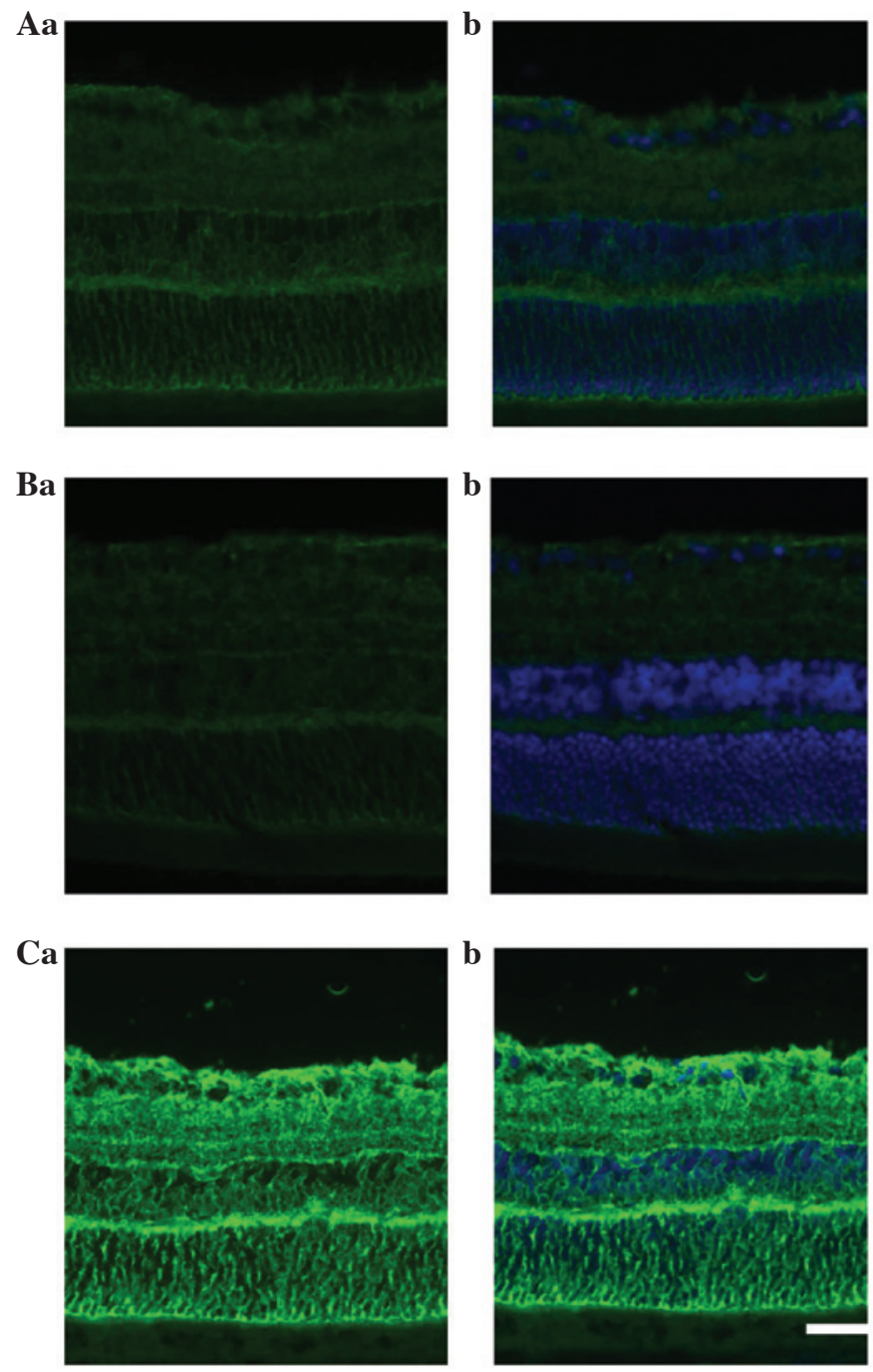

Figure 3. Immunofluorescence images of adenosine- and adenosine antagonist-induced changes in GLAST expression. Adenosine and/or SCH442416 were injected into the vitreous space 2 weeks before the surgery. (A-C) Immunofluorescence labeling showing the (Aa, Ba and Ca) GLAST protein expression and $(\mathrm{Ab}, \mathrm{Bb}$ and $\mathrm{Cb})$ GLAST with DAPI in retinal slices taken from the (A) control, (B) adenosine-injected or (C) adenosine + SCH442416-injected rat eyes. Treatment with adenosine decreased the GLAST protein expression compared with the control, while the administration of adenosine + SCH442416 markedly increased GLAST fluorescence compared with the control and adenosine groups $(\mathrm{n}=6)$. Scale bar, $20 \mu \mathrm{m}$. GLAST, L-glutamate/L-aspartate transporter.

Adenosine- and adenosine $A_{2 A}$ receptor antagonist-induced changes in GLAST mRNA expression in the rat $\mathrm{COH}$ models. Adenosine or adenosine + SCH442416 solution was intravitreally injected into the right eye of the rats. The rats were sacrificed two weeks later by cervical dislocation and GLAST mRNA expression was evaluated through RT-qPCR analysis. Compared with the control $(\mathrm{COH})$ group, the GLAST mRNA expression was observed to decrease by $33.6 \%$ in the adenosine treatment group $(n=6, P=0.020)$ and to increase by $159.6 \%$ in the group treated with $\mathrm{SCH} 442416(\mathrm{n}=6, \mathrm{P}=0.001)$ (Fig. 1).

Adenosine-and adenosine $A_{2 A}$ receptor antagonist-induced changes in GLAST protein expression in the rat $\mathrm{COH}$ models. Adenosine or adenosine + SCH442416 solution was intravitreally injected into the right eye of the rats. The rats were 
sacrificed two weeks later, and the retinas were dissected and used for the experiment. Compared with the control $(\mathrm{COH})$ group, the administration of adenosine decreased GLAST protein expression in the retina by $34.7 \%(n=6, P<0.001)$, whereas treatment with the adenosine $\mathrm{A}_{2 \mathrm{~A}}$ receptor antagonist SCH442416 increased GLAST protein expression by $48.3 \%$ $(n=6, P<0.001)$ (Fig. 2A and B).

In the immunohistochemical experiments, the administration of adenosine was shown to decrease GLAST protein expression relative to that in the control rat retina. By contrast, the administration of SCH442416 markedly increased the GLAST protein expression compared with that in the control and adenosine groups (Fig. 3A-C).

\section{Discussion}

Retinal Müller cells release adenosine and glutamate in pathological conditions. Glutamate is a major excitatory neurotransmitter in the mammalian retina and its presence at high concentrations plays a key role in neuronal damage. Extracellular glutamate is primarily taken up into the cell via GLAST ( $50 \%$ of extracellular glutamate) (11); however, the effect of adenosine on GLAST function and the underlying mechanism have yet to be fully elucidated. The results of the present study have provided the first evidence, to the best of our knowledge, that treatment with adenosine $(10 \mu \mathrm{M})$ can decrease the retinal GLAST protein and mRNA expression. It has been reported that elevations in IOP to $<70 \mathrm{mmHg}$ are conducive to the maintenance of GLAST activity in rats (12). The present study results were consistent with this finding. Decreases in the expression of GLAST protein were accompanied by decreases in the GLAST mRNA level, suggesting that decreased transcription plays an important role in the downregulation of GLAST protein expression.

Adenosine, one of the most bioactive substances, is widely present in intracellular and extracellular fluid (13). Adenosine produces its biological effect through adenosine receptors. All adenosine receptors are $\mathrm{G}$-protein-coupled receptors and can be grouped into four subtypes: $A_{1}, A_{2 A}, A_{2 B}$ and $A_{3}$. Biological and pharmacological studies have found that all four adenosine receptor are located in the retina. The $A_{1}$ receptor $\left(A_{1} R\right), A_{2 A} R$ and $A_{2 B} R$ have been studied extensively, while less focus has been placed on the $A_{3} R$. It has been demonstrated that $A_{1} R$ activation has a protective function in vivo, inhibiting inflammation and apoptosis in the heart, brain and kidneys (14). The release of neurotransmitters, including glutamate, from the synaptic terminals is inhibited by activation of the $A_{1} R$. By contrast, activation of the $A_{2 A} R$ promotes the release of neurotransmitters, including glutamate. The $\mathrm{A}_{2 \mathrm{~B}} \mathrm{R}$ is expressed at a low density in almost all tissues and exhibits low-affinity ligand binding (15). Less is known about the $A_{3} R$ than about the $A_{1} R, A_{2 A} R$ and $A_{2 B} R$. With regard to the $A_{2 A} R$, early studies have indicated that $\mathrm{A}_{2 \mathrm{~A}} \mathrm{R}$ antagonists can induce a decrease in glutamate uptake in a mouse model of Alzheimer's disease $(15,16)$. In the present study, an $\mathrm{A}_{2 \mathrm{~A}} \mathrm{R}$ antagonist was selected for the study, since none of the other adenosine receptors tested $\left(A_{1} R\right.$ or $\left.A_{3} R\right)$ modified glutamate uptake. Although a number of previous studies have shown that $A_{2 A} R$ antagonists increase GLAST expression in cultured cells $(17,18)$, the results of the present study provide the first evidence, to the best of our knowledge, that the blockage of $\mathrm{A}_{2 \mathrm{~A}} \mathrm{Rs}$ can increase the GLAST expression in the rat retina in vivo. The results of the present study may provide a novel method for retinal neuron protection.

\section{Acknowledgements}

This study was funded by the National Natural Science Foundation of China (grant no. 81371014) and the Shanghai 'Science and Technology Innovation Action Plan' Basic Research Key Project (grant nos. 11JC1407700 and 11JC1407701).

\section{References}

1. Linnertz R, Wurm A, Pannicke T, et al: Activation of voltage-gated $\mathrm{Na}^{+}$and $\mathrm{Ca}^{2+}$ channels is required for glutamate release from retinal glial cells implicated in cell volume regulation. Neuroscience 188: 23-34, 2011

2. Brückner E, Grosche A, Pannicke T, Wiedemann P, Reichenbach A and Bringmann A: Mechanisms of VEGF- and glutamate-induced inhibition of osmotic swelling of murine retinal glial (Müller) cells: Indications for the involvement of vesicular glutamate release and connexin-mediated ATP release. Neurochem Res 37: 268-278, 2012.

3. Mubagwa K and Flameng W: Adenosine, adenosine receptors and myocardial protection: An updated overview. Cardiovasc Res 52: 25-39, 2001.

4. Ribelayga $\mathrm{C}$ and Mangel SC: A circadian clock and light/dark adaptation differentially regulate adenosine in the mammalian retina. $\mathrm{J}$ Neurosci 25: 215-222, 2005.

5. Clark BD, Kurth-Nelson ZL and Newman EA: Adenosine-evoked hyperpolarization of retinal ganglion cells is mediated by G-protein-coupled inwardly rectifying $\mathrm{K}^{+}$and small conductance $\mathrm{Ca}^{+}$-activated $\mathrm{K}^{+}$channel activation. J Neurosci 29: 11237-11245, 2009.

6. Hertz L: The glutamate-glutamine (GABA) cycle: Importance of late postnatal development and potential reciprocal interactions between biosynthesis and degradation. Front Endocrinol (Lausanne) 4: 59, 2013.

7. Reichenbach A and Bringmann A: New functions of Müller cells. Glia 61: 651-678, 2013.

8. Isbikawa $\mathrm{M}$, Yoshitomi $\mathrm{T}$, Zorumski $\mathrm{CF}$ and Izumi $\mathrm{Y}$ : Downregulation of glutamine synthetase via GLAST suppression induces retinal axonal swelling in a rat ex vivo hydrostatic pressure model. Invest Ophthalmol Vis Sci 52: 6604-6616, 2011.

9. Shareef SR, Garcia-Valenzuela E, Salierno A, Walsh J and Sharma SC: Chronic ocular hypertension following episcleral venous occlusion in rats. Exp Eye Res 61: 379-382, 1995.

10. Chen J, Miao Y, Wang XH and Wang Z: Elevation of p-NR2A(S1232) by $\mathrm{Cdk} 5 / \mathrm{p} 35$ contributes to retinal ganglion cell apoptosis in a rat experimental glaucoma model. Neurobiol Dis 43: 455-464, 2011.

11. Sarthy VP, Pignataro L, Pannicke T, et al: Glutamate transport by retinal Müller cells in glutamate/aspartate transporter-knockout mice. Glia 49: 184-196, 2005.

12. Holcombe DJ, Lengefeld N, Gole GA and Barnett NL: The effects of acute intraocular pressure elevation on rat retinal glutamate transport. Acta Ophthalmol 86: 408-414, 2008.

13. Zhong Y, Yang Z, Huang WC and Luo X: Adenosine, adenosine receptors and glaucoma: An updated overview. Biochim Biophys Acta 1830: 2882-2890, 2013.

14. Kim M, Chen SW, Park SW, et al: Kidney-specific reconstitution of the A1 adenosine receptor in A1 adenosine receptor knockout mice reduces renal ischemia-reperfusion injury. Kidney Int 75: 809-823, 2009.

15. Stone TW, Ceruti S and Abbracchio MP: Adenosine receptors and neurological disease: Neuroprotection and neurodegeneratioon. Handb Exp Pharmacol 193: 535-587, 2009.

16. Takahashi RN, Pamplona FA and Prediger RD: Adenosine receptor antagonists for cognitive dysfunction: A review of animal studies. Front Biosci 13: 2614-2632, 2008.

17. Yu J, Zhong Y, Shen X, Cheng Y, Qi J and Wang J: In vitro effect of adenosine A2A receptor antagonist SCH 442416 on the expression of glutamine synthetase and glutamate aspartate transporter in rat retinal Müller cells at elevated hydrostatic pressure. Oncol Rep 27: 748-752, 2012.

18. Valadas JS, Batalha VL, Ferreira DG, et al: Neuroprotection afforded by adenosine A2A receptor blockade is modulated by corticotrophin-releasing factor (CRF) in glutamate injured cortical neurons. J Neurochem 123: 1030-1040, 2012. 